\title{
Experiments with mid-heavy antiprotonic atoms in AEgIS
}

\author{
G. Kornakov, ${ }^{a, *}$ M. Auzins, ${ }^{b}$ B. Bergmann, ${ }^{c}$ P. Burian, ${ }^{c}$ G. Bonomi, ${ }^{d, e}$ R. S. Brusa,,${ }^{f, g}$ \\ A. Camper, ${ }^{h}$ R. Caravita, ${ }^{f, g}$ F. Castelli, ${ }^{i, j}$ P. Cheinet, ${ }^{k}$ R. Ciuryło, ${ }^{l}$ D. Comparat, ${ }^{k}$ \\ G. Consolati, ${ }^{i, m}$ M. Doser, ${ }^{n}$ H. Gjersdal, ${ }^{h}$ L. T. Glöggler, ${ }^{n}$ Ł. Graczykowski, ${ }^{a}$ \\ F. Guatieri, ${ }^{f, g}$ S. Haider, ${ }^{n}$ S. Huck, ${ }^{n}$ M. Janik, ${ }^{a}$ G. Kasprowicz, ${ }^{a}$ G. Khatri, ${ }^{, n}$ \\ Ł. Kłosowski, ${ }^{l}$ L. Lappo, ${ }^{a}$ C. Malbrunot, ${ }^{n, o}$ S. Mariazzi, ${ }^{g, f}$ G. Nebbia, ${ }^{p}$ L. Nowak, ${ }^{n}$ \\ D. Nowicka, ${ }^{a}$ E. Oswald, ${ }^{n}$ D. Pagano, ${ }^{d, e}$ L. Penasa, ${ }^{f, g}$ V. Petracek, ${ }^{c}$ M. Piwiński, ${ }^{l}$ \\ S. Pospisil, ${ }^{c}$ L. Povolo, ${ }^{g, f}$ F. Prelz, ${ }^{i}$ S. Rangwala, ${ }^{q}$ B. Rienäcker, ${ }^{n}$ A. Rotondi, ${ }^{e, r}$ \\ O. M. Røhne, ${ }^{h}$ H. Sandaker, ${ }^{h}$ I. Stekl, ${ }^{c}$ D. Tefelski, ${ }^{a}$ I. C. Tietje, ${ }^{n}$ M. Volponi, ${ }^{f, g, n}$ \\ T. Wolz, ${ }^{n}$ M. Zawada, ${ }^{l}$ C. Zimmer ${ }^{h, m, n}$ and N. Zurlo ${ }^{e, s}$
}

E-mail: georgy.kornakov@pw. edu.pl

\begin{abstract}
Antiprotonic atoms have been fundamental in experiments which provide the most precise data on the strong interaction between protons and antiprotons and of the neutron skin of many nuclei thanks to the clean annihilation signal. In most of these experiments, the capture process of low energy antiprotons was done in a dense target leading to a significant suppression of specific transitions between deeply bound levels that are of particular interest. In particular, precise measurements of specific transitions in antiprotonic atoms with $\mathrm{Z}>2$ are sparse.

We propose to use the pulsed production scheme developed for antihydrogen and protonium for the formation of cold antiprotonic atoms. This technique has been recently achieved experimentally for the production of antihydrogen at AEḡIS. The proposed experiments will have sub-ns synchronization thanks to an improved control and acquisition system. The formation in vacuum guarantees the absence of Stark mixing or annihilation from high $\mathrm{n}$ states and together with the sub-ns synchronization would resolve the previous experimental limitations. It will be possible to access the whole chain of the evolution of the system from its formation until annihilation with significantly improved signal-to-background ratio.
\end{abstract}

\footnotetext{
*** Particles and Nuclei International Conference - PANIC2021 ***

*** 5 - 10 September, 2021 ***

$* * *$ Online $* * *$
}

${ }^{*}$ Speaker 


\section{Introduction}

Exotic bound systems similar to normal atoms can be created if one of the sub-atomic particles is substituted by another of a different type but with equal charge. Negatively-charged particles can substitute the electrons and neutral or positive can replace the nucleons. The antiproton has the same electric charge as the electron and thus can replace one of them in an atom forming a special type of exotic atoms called antiprotonic atoms. The antiprotons are as stable as their matter partners, the protons. After the capture of an antiproton, the atom will transit from an excited state towards annihilation on the surface of the nucleus, ejecting in the process all or almost all electrons via Auger emission. These atoms have been fundamental to establish the distribution of neutrons in nuclei [1], the strong interaction between antiprotons and protons [2], and tests of quantum theories [3] by observing the annihilation signals and the energy and width of the transitions.

However, most of the previous studies have been made with $\mathrm{MeV}$ antiprotons in bulk matter and precise spectroscopic measurements of the atomic levels are missing for $\mathrm{Z}>2$. Here, we propose a novel scheme for formation of antiprotonic atoms from trapped atoms within the Penning-Malmberg trap of the AEḡIS (Antimatter Experiment: Gravity, Interferometry, Spectroscopy) apparatus [4] located at the Antiproton Decelerator (AD) facility at CERN [5].

\section{The AEgIS experiment and apparatus}

At present, the Antiproton Decelerator facility is the world's only source of abundant antimatter and no others are expected to operate at least until the start of the antiproton program at FAIR, in Germany, beyond 2025 [6]. The AD facility provides access to antiprotons produced in collisions of a $20 \mathrm{GeV}$ proton beam impinging on an Iridium target after which they are decelerated from relativistic energies down to $100 \mathrm{keV}$ in two rings by means of electron and sympathetic cooling stages. The first ring is the $\mathrm{AD}$ itself, which reduces the energy of the antiprotons to $5.3 \mathrm{MeV}$. The second one, ELENA (The Extra Low Energy Antiproton ring) [5], is a small $30 \mathrm{~m}$ circumference synchrotron that brings the energy down to $100 \mathrm{keV}$ and finally transfers them to the experiments present at the antimatter laboratory.

One of them is the AEgIS apparatus. It implements two cylindrical cryostats containing $5 \mathrm{~T}$ and a $1 \mathrm{~T}$ superconducting magnets, which surround the regions of antiproton trapping and formation of antihydrogen. A series of cylindrical electrodes form a Penning-Malmberg trap arrangement and provide radial and axial confinement of the plasma of charged particles. An external positron source is available for pulsed production of Positronium (Ps), a type of exotic atom formed by an electron and a positron. The manipulation of the states is achieved by synchronised pulsed laser radiation that can be directed to the production zone. A set of detectors allow one to monitor and control the plasmas and the antihydrogen formation process. The pulsed scheme for resonant production of antihydrogen via Rydberg Ps and trapped cold antiprotons is shown in Figure 1.

\section{Upgrade of the control system}

Such an experiment requires a complex control system that provides a precise control of electrodes, photonic circuits, plasma and beam diagnostics, time synchronization, state readout and 


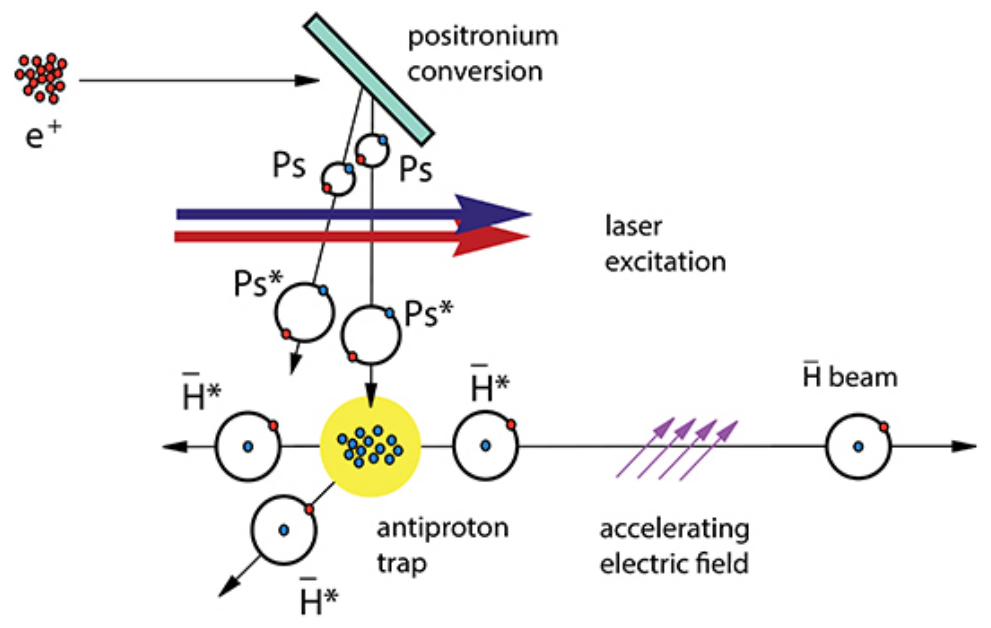

Figure 1: Schematic representation of the pulsed production of an antihydrogen beam from resonant exchange reaction between Rydberg positronium and trapped antiprotons in a Malmberg-Penning trap.
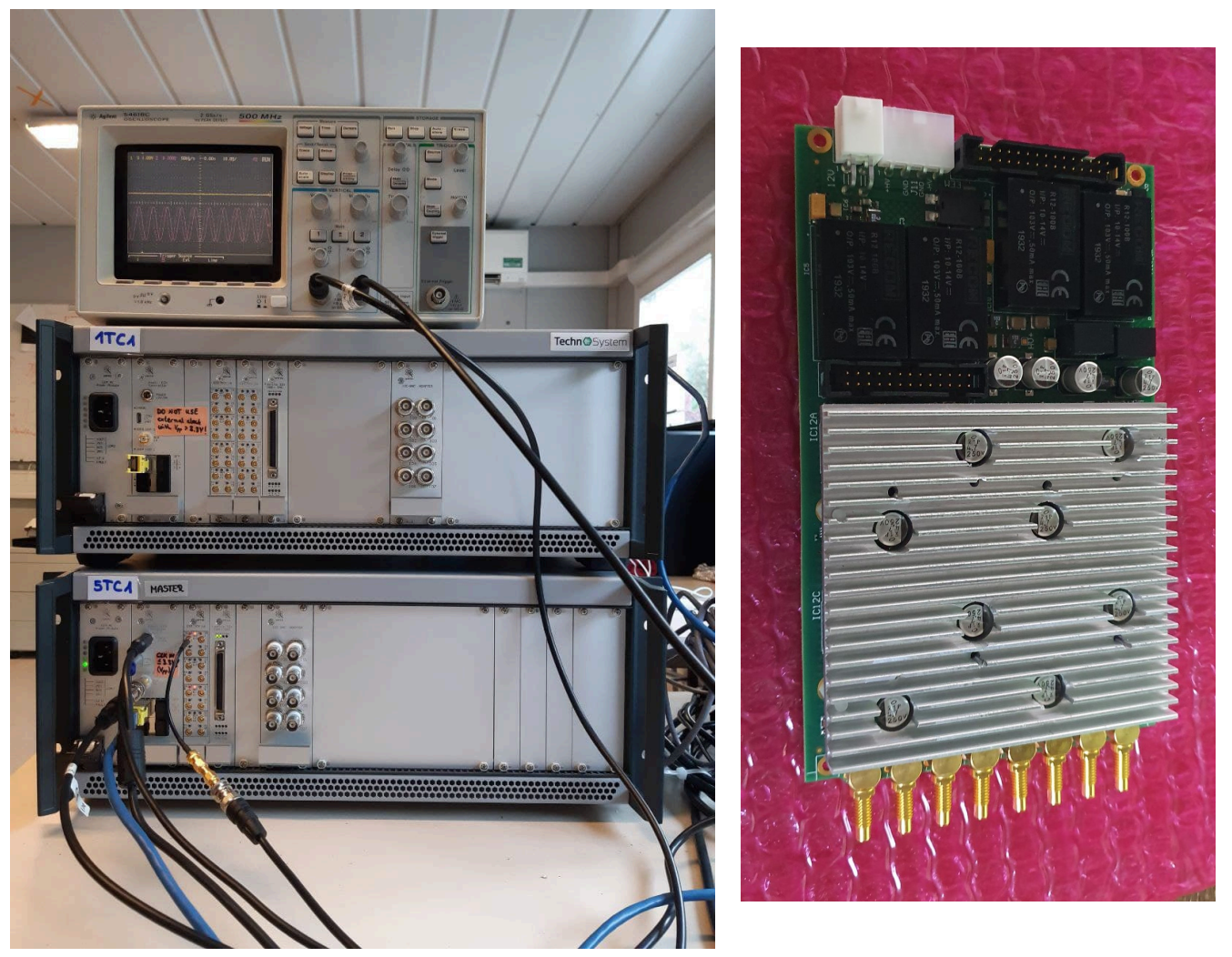

Figure 2: Right Test bench of the control electronics based on Sinara/ARTIQ for the 1T and $5 \mathrm{~T}$ traps consisting of a Kasli (a FPGA carrier used as the ARTIQ central core device), Fastino (Fast 32-channel 16bit DAC EEM), DIO MCX 16CH and PSU modules. Left High Voltage amplifiers which boosts the Fastino output to $\pm 200 \mathrm{~V}$. 


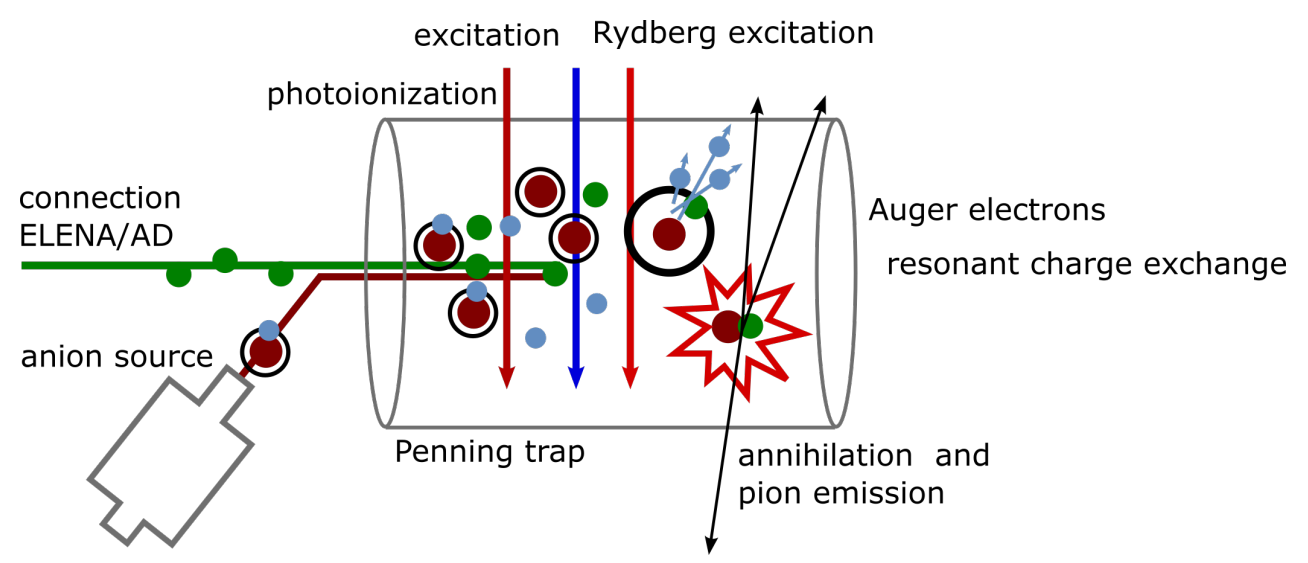

Figure 3: Schematic representation of the formation of antiprotonic atoms inside the Penning-Malmberg trap. The negative ions, shown as red circles with an additional electron in blue, produced in an external source are brought inside the main apparatus. After accumulating together with antiprotons, shown as green circles, three synchronized lasers first photo-detach the electrons, and then excite the neutral atoms to the first and to a highly excited Rydberg state. At this moment a resonant exchange reaction happens between the Rydberg atoms and the antiprotons, forming the antiprotonic atoms. After approximately $10 \mathrm{~ns}$ the antiprotons reach the surface of the nuclei and annihilate.

a flexible and easy to use user interface. These requirements are met by a system like Sinara [7]. Sinara is an open-source (CERN Open Hardware Licence v1.2) hardware ecosystem for AMO laboratories mainly focused on experiments with trapped ions and quantum technologies. The system allows one to operate, monitor and control the data acquisition system, high voltage of ion traps, lasers, detectors, optical elements, magnets, radio frequency generators and beam diagnostics. Sinara is deployed with ARTIQ (Advanced Real-Time Infrastructure for Quantum physics) control software [8]. The control is implemented on FPGA technology and offers a sub-ns time distribution to all subsystems of the experiment.

The experimental requirements of AEḡIS demanded a new High Voltage amplifier board to control the trap electrodes with 8 independent channel. The board provides $\pm 200 \mathrm{~V}$ range, $1 \mathrm{MHz}$ bandwidth, $50 \mathrm{Ohm}$ output impedance, overheating protection and a quick output disconnect controlled via EEM using OptoMos to minimise the noise in the trapping electrodes. The crates with control electronics for the traps and the amplification board are shown in Figure 2.

\section{Pulsed production scheme of antiprotonic atoms}

We propose to adapt an already developed scheme [9] for the pulsed production of highly excited atoms of protonium ( $\mathrm{Pn}$ ), a bound state of a proton and an antiproton, to the production of any antiprotonic atom starting from a trapped negative ion of tens of meV. The scheme relies on the resonant-charge-exchange reaction:

$$
A^{*}+\bar{p} \rightarrow \bar{p} A^{*}+e^{-},
$$

where a Rydberg excited atom $A^{*}$ and antiprotons interact to form the antiprotonic atom $\bar{p} A^{*}$ as shown in Figure 3. $A^{*}$ is created from pulsed laser photo-detached and excited anions $\left(A^{-}\right)$, which 
are initially trapped and stored in a plasma together with other negative particles such as electrons and antiprotons. After that, a charge exchange reaction between the Rydberg atoms $\mathrm{A}^{*}$ and the antiprotons occurs. For light and mid-heavy atoms with electron binding energies below $40 \mathrm{keV}$, the stripping of electrons is complete at the moment of annihilation [1]. This process lasts for approximately $10 \mathrm{~ns}$ [10], finishing with the annihilation of the antiproton on the surface of the nuclei that can be precisely synchronized to the formation time using the upgraded control scheme providing background-free access to the formation and annihilation processes.

\section{Summary}

The availability of low energy antiprotons from ELENA enables experiments to provide the most precise tests of theories on antimatter. Combining the unique features of AE $\bar{g} I S$ an accurate measurement of antiprotonic atoms can be performed from the exited Rydberg states until the annihilation on the surface.

\section{Acknowledgements}

This research was funded by Warsaw University of Technology within the Excellence Initiative: Research University (IDUB) programme and the IDUB-POB-FWEiTE-1 project grant.

\section{References}

[1] G. Backenstoss, Antiprotonic atoms, Contemp. Phys. 30 (1989) 433.

[2] E. Klempt, F. Bradamante, A. Martin and J. Richard, Anti-nucleon-nucleon interaction at low energy: scattering and protonium, Phys. Rept. 368 (2002) 119.

[3] M.S. Safronova, D. Budker, D. DeMille, D.F.J. Kimball, A. Derevianko and C.W. Clark, Search for New Physics with Atoms and Molecules, Rev. Mod. Phys. 90 (2018) 025008 [1710.01833].

[4] C. Amsler et al., Pulsed production of antihydrogen, Commun. Phys. 4 (2021) 19.

[5] ELENA, AD collaboration, The ELENA facility, Phil. Trans. Roy. Soc. Lond. A 376 (2018) 20170266.

[6] PANDA collaboration, Physics Performance Report for PANDA: Strong Interaction Studies with Antiprotons, 0903.3905.

[7] "Sinara open-source hardware ecosystem." https://github.com/sinara-hw/meta/wiki.

[8] "Artiq (advanced real-time infrastructure for quantum physics)." https://m-labs.hk/artiq/.

[9] S. Gerber, M. Doser and D. Comparat, Pulsed production of cold protonium in Penning traps, Phys. Rev. A 100 (2019) 063418.

[10] D. Gotta, Precision spectroscopy of light exotic atoms, Prog. Part. Nucl. Phys. 52 (2004) 133. 


\section{Experiments with mid-heavy antiprotonic atoms in AEgIS}

\section{The AĒ̄IS Collaboration}

${ }^{a}$ Warsaw University of Technology, Faculty of Physics, ul. Koszykowa 75, 00-662, Warsaw, Poland

${ }^{b}$ University of Latvia, Department of Physics, Raina boulevard 19, LV-1586, Riga, Latvia

${ }^{c}$ Czech Technical University, Prague, Brehová 7, 11519 Prague 1, Czech Republic

${ }^{d}$ Department of Mechanical and Industrial Engineering, University of Brescia, via Branze 38, 25123 Brescia, Italy

e INFN Pavia, via Bassi 6, 27100 Pavia, Italy

${ }^{f}$ Department of Physics, University of Trento, via Sommarive 14, 38123 Povo, Trento, Italy

${ }^{g}$ TIFPA/INFN Trento, via Sommarive 14, 38123 Povo, Trento, Italy

${ }^{h}$ Department of Physics, University of Oslo, Sem Salandsvei 24, 0371 Oslo, Norway

${ }^{i}$ INFN Milano, via Celoria 16, 20133 Milano, Italy

${ }^{j}$ Department of Physics “Aldo Pontremoli”, University of Milano, via Celoria 16, 20133 Milano, Italy

${ }^{k}$ Laboratoire Aimé Cotton, Université Paris-Sud, ENS Paris Saclay, CNRS, Université Paris-Saclay, 91405 Orsay Cedex, France

${ }^{l}$ Institute of Physics, Faculty of Physics, Astronomy, and Informatics, Nicolaus Copernicus University in Torun, Grudziadzka 5, 87-100 Toruń, Poland

${ }^{m}$ Department of Aerospace Science and Technology, Politecnico di Milano, via La Masa 34, 20156 Milano, Italy

${ }^{n}$ Physics Department, CERN, 1211 Geneva 23, Switzerland

${ }^{o}$ Stefan Meyer Institute for Subatomic Physics, Austrian Academy of Sciences, Boltzmanngasse 3, 1090 Vienna, Austria

PINFN Padova, via Marzolo 8, 35131 Padova, Italy

${ }^{q}$ Raman Research Institute, C. V. Raman Avenue, Sadashivanagar, Bangalore 560080, India

${ }^{r}$ Department of Physics, University of Pavia, via Bassi 6, 27100 Pavia, Italy

${ }^{s}$ Department of Civil, Environmental, Architectural Engineering and Mathematics, University of Brescia, via Branze 43, 25123 Brescia, Italy

\footnotetext{
*** Particles and Nuclei International Conference - PANIC2021 ***

*** 5 - 10 September, $2021 * * *$

$* * *$ Online $* * *$
} 